\title{
Prognosis of Extracapsular Spread of Cervical Lymph Node Metastases in Nasopharyngeal Carcinoma
}

\author{
Xue Yin ${ }^{1 \dagger}$, Lu Lv' ${ }^{1 \dagger}$ and Xin-Bin Pan ${ }^{2 *}$ \\ 'Department of Oncology, The Central Hospital of Wuhan, Tongji Medical College, Huazhong University of Science \\ and Technology, Wuhan, China, ${ }^{2}$ Department of Radiation Oncology, Guangxi Medical University Cancer Hospital, Nanning, \\ China
}

Purpose: This study aims to identify the prognosis of the extracapsular spread (ECS) of cervical lymph node metastases in nasopharyngeal carcinoma (NPC).

Materials and Methods: Patients with NPC were extracted from the Surveillance, Epidemiology, and End Results (SEER) database from 2004 to 2016. Pathologically confirmed World Health Patients with World Health Organization types I, II, and III NPC with complete ECS data of cervical lymph node metastases were investigated. The

OPEN ACCESS

Edited by:

Paolo Bossi,

University of Brescia, Italy

Reviewed by:

XiaoShen Wang,

Fudan University, China Nicola Alessandro lacovelli, Istituto Nazionale dei Tumori (IRCCS),

Italy

*Correspondence:

Xin-Bin Pan

panxinbin@gxmu.edu.cn

panxinbin@aliyun.com

†These authors have contributed equally to this work

Specialty section:

This article was submitted to Head and Neck Cancer,

a section of the journal

Frontiers in Oncology

Received: 01 January 2020

Accepted: 28 August 2020 Published: 25 September 2020

Citation:

Yin X, Lv L and Pan X-B (2020) Prognosis of Extracapsular Spread of Cervical Lymph Node Metastases

in Nasopharyngeal Carcinoma.

Front. Oncol. 10:523956.

doi: 10.3389/fonc.2020.523956 included patients were divided into non-ECS and ECS groups. The 10-year overall survival (OS) and cancer-specific survival (CSS) were compared between the two groups using the Kaplan-Meier method and propensity score matching analyses.

Results: A total of 625 patients were included. The ECS group included 99 (15.84\%) patients. The non-ECS group included 526 (84.16\%) patients. The 10-year OS $(50.2$ vs. $35.8 \% ; P<0.001$ ) and CSS (64.8 vs. $45.7 \%$; $P<0.001$ ) were better in the nonECS group than in the ECS group in the unmatched cohort. Propensity score matching analyses revealed favorable 10-year OS (52.7 vs. 35.8\%; $P=0.008$ ) and CSS (61.2 vs. $45.7 \% ; P=0.008$ ) in the non-ECS group with respect to the ECS group. Age, sex, race, AJCC stage, and ECS (hazard ratio $(\mathrm{HR})=1.71$, 95\% confidence interval $(\mathrm{Cl}), 1.14-$ 2.57, $P=0.010$ ) were independent prognostic factors for OS. Age, sex, AJCC stage, and $\mathrm{ECS}(\mathrm{HR}=1.91 ; 95 \% \mathrm{Cl}, 1.21-3.01 ; P=0.005)$ were independent prognostic factors for CSS.

Conclusion: This study indicated that ECS is a prognostic risk factor for NPC. Further studies should be performed to verify the results due to the limitations of the SEER database.

Keywords: nasopharyngeal carcinoma, extracapsular spread, NPC, ECS, prognosis

\section{INTRODUCTION}

Nasopharyngeal carcinoma (NPC) is a highly epidemiological cancer in South China $(1,2)$. It is a unique head and neck cancer with a high incidence of cervical lymph node metastases. Approximately $85 \%$ of patients present with cervical lymph node metastases at diagnosis (3). Moreover, extracapsular spread (ECS) has been found in $33.6-75.6 \%$ of patients with cervical lymph node metastases (4-6). In other head and neck cancers, ECS has been suggested to be a risk prognostic factor $(7,8)$. However, the prognosis of the ECS of cervical lymph node metastases 
in NPC has not been well investigated. Mao et al. (4) revealed that ECS was an independent risk prognostic factor. In contrast, several studies reported that ECS was not associated with survival $(5,6,9)$. To identify the prognosis of patient with NPC with ECS of cervical lymph node metastases, this retrospective cohort study was conducted using data from the Surveillance, Epidemiology, and End Results (SEER) database.

\section{MATERIALS AND METHODS}

\section{Patients}

Patients with NPC from the SEER database between 2004 and 2016 were investigated. The inclusion criteria were as follows: (1) patients with pathologically confirmed NPC; (2) patients with World Health Organization (WHO) type I, II, or III NPC; (3) patients with definite American Joint Committee on Cancer (AJCC) TNM stages; (4) patients with a definite tumor grade; and (5) patients with definite information on the ECS of cervical lymph node metastases. Included patients were divided into ECS and non-ECS groups.

According to the AJCC, ECS is assessed by physical examination and imaging [computed tomography (CT) scan or magnetic resonance imaging (MRI)] (10). ECS can be diagnosed clinically by a matted mass of nodes adherent to overlying skin or adjacent soft tissue or clinical evidence of cranial nerve invasion. The criteria of radiological signs of ECS were as follows: (1) amorphous and speculated margins of a metastatic node; (2) the presence of indistinct nodal margins, irregular nodal capsular enhancement, or infiltration into the adjacent fat or muscle; and (3) fusion status of metastatic nodes (11-13). In the SEER database, ECS was coded as "001," while non-ECS was coded as "000" under the variable "CS Site-Specific Factor 2."

\section{Endpoints}

The primary endpoint was overall survival (OS). OS was defined in the SEER database as the time from diagnosis to death as a result of any cause. The secondary endpoint was cancer-specific survival (CSS). CSS was defined as the time from diagnosis to death attributed to NPC.

\section{Statistical Analysis}

The continuous variable of age was transformed into a categorical variable (14). Categorical variables, including age, sex, race, tumor grade, tumor pathology, AJCC stage, T stage, N stage, $M$ stage, radiotherapy, and chemotherapy, between the non-ECS and ECS groups were analyzed by using the $\chi^{2}$ test or Fisher's exact test. Logistic regression analysis was performed to identify factors associated with ECS.

The 10-year OS and CSS rates of the ECS and non-ECS groups were calculated using Kaplan-Meier analysis. The survival difference between the ECS and non-ECS groups was examined by the log-rank test. Multivariable proportional hazards models adjusted for age, sex, race, tumor grade, tumor pathology, AJCC stage, radiotherapy, and chemotherapy were implemented to assess independent prognostic factors. The results are reported as hazard ratios (HRs) with 95\% confidence intervals (CIs).
A matched case-control analysis was conducted by using propensity score matching (PSM) to reduce the influence of selection bias on the comparison between ECS and non-ECS groups. A logistic regression model was established in which ECS status was taken as the dependent variable in the process of calculating the propensity scores. One-to-one matching without replacement was performed using the nearest-neighbor match on the logit of the propensity score for confounding factors (derived from age, sex, race, tumor grade, tumor pathology, AJCC stage, radiotherapy, and chemotherapy). Standard differences for each of the covariates were used to compare the similarity of ECS and non-ECS groups after matching. An absolute value $<0.05$ indicates that covariates are well balanced in both groups (15).

SPSS Statistics Version 26.0 software (IBM Co., Armonk, NY, United States) and R software (version 3.6.2) were used to perform statistical analyses. Two-tailed $P<0.05$ was considered statistically significant.

\section{RESULTS}

\section{Patient Characteristics}

Figure 1 shows the process of patient selection. This study finally included 625 patients. The ECS group included 99 (15.84\%) patients. The non-ECS group included 526 (84.16\%) patients. Table 1 shows the patient characteristics. The median follow-up times were 85 months (interquartile range (IQR),

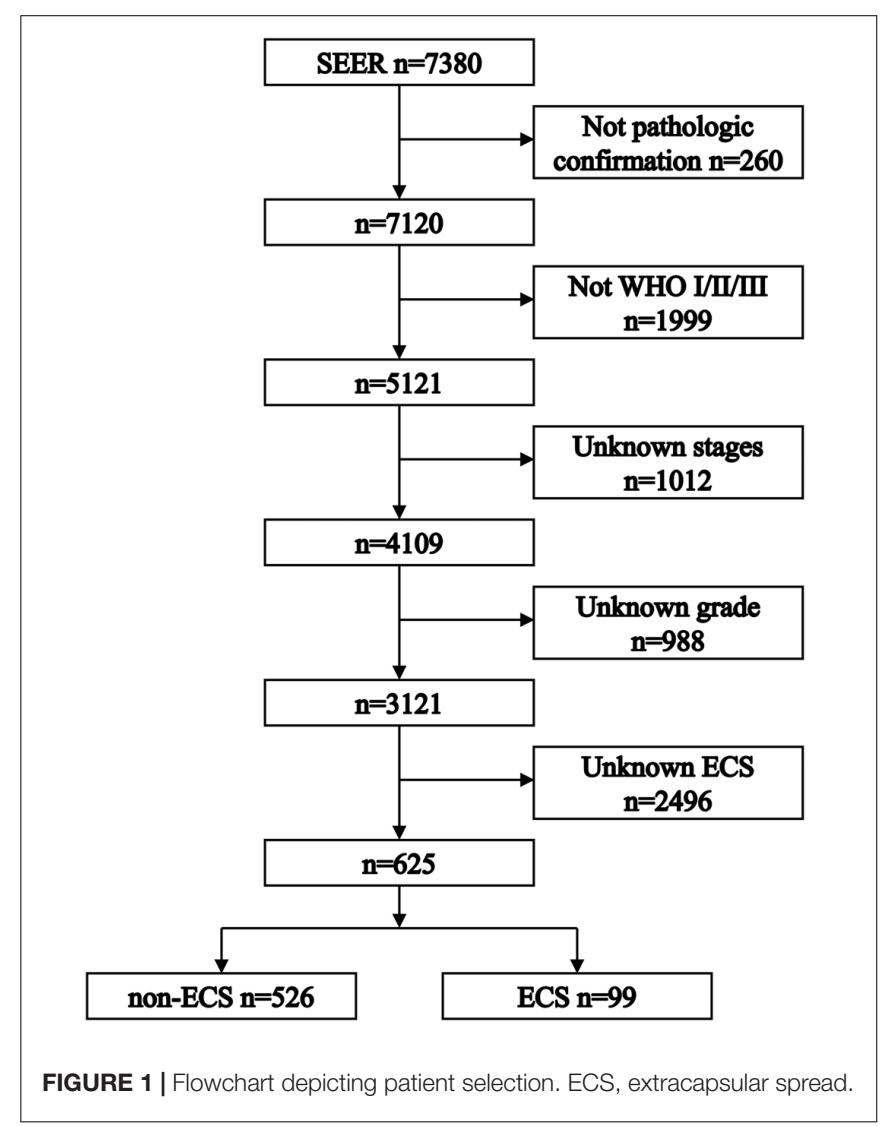


TABLE 1 | Patient characteristics.

\begin{tabular}{|c|c|c|c|c|}
\hline & Total $(n=625)$ & Non-ECS $(n=526)$ & $\operatorname{ECS}(n=99)$ & $P$ value \\
\hline$\leq 19$ & 23 (3.68\%) & 18 (3.42\%) & $5(5.05 \%)$ & Reference \\
\hline $40-59$ & $326(52.16 \%)$ & 285 (54.18\%) & $41(41.41 \%)$ & 0.209 \\
\hline $60-79$ & $171(27.36 \%)$ & 135 (25.67\%) & $36(36.36 \%)$ & 0.940 \\
\hline$\geq 80$ & 15 (2.40\%) & $12(2.28 \%)$ & 3 (3.03\%) & 0.898 \\
\hline Female & 165 (26.40\%) & 147 (27.95\%) & $18(18.18 \%)$ & 0.043 \\
\hline Male & 460 (73.60\%) & 379 (72.05\%) & 81 (81.82\%) & \\
\hline \multicolumn{5}{|l|}{ Race } \\
\hline Asian & 238 (38.08\%) & 207 (39.35\%) & 31 (31.31\%) & Reference \\
\hline Black & $74(11.84 \%)$ & 59 (11.22\%) & $15(15.15 \%)$ & 0.125 \\
\hline White & $313(50.08 \%)$ & 260 (49.43\%) & $53(53.54 \%)$ & 0.206 \\
\hline III & 269 (43.04\%) & 226 (42.97\%) & $43(43.43 \%)$ & 0.856 \\
\hline IV & 259 (41.44\%) & 222 (42.21\%) & 37 (37.37\%) & 0.703 \\
\hline \multicolumn{5}{|c|}{ Pathology } \\
\hline WHOI & 267 (42.72\%) & 218 (41.44\%) & 49 (49.49\%) & Reference \\
\hline WHO ॥ & $160(25.60 \%)$ & $135(25.67 \%)$ & 25 (25.25\%) & 0.471 \\
\hline WHO III & $198(31.68 \%)$ & 173 (32.89\%) & 25 (25.25\%) & 0.095 \\
\hline \multicolumn{5}{|c|}{ AJCC stage } \\
\hline$\|$ & 190 (30.40\%) & 171 (32.51\%) & 19 (19.19\%) & Reference \\
\hline III & 234 (37.44\%) & 206 (39.16\%) & $28(28.28 \%)$ & 0.521 \\
\hline $\mathrm{IVa}$ & 58 (9.28\%) & 47 (8.94\%) & $11(11.11 \%)$ & 0.067 \\
\hline $\mathrm{IVb}$ & 79 (12.64\%) & $58(11.03 \%)$ & $21(21.21 \%)$ & 0.001 \\
\hline $\mathrm{N} 1$ & 298 (47.68\%) & 263 (50.00\%) & 35 (35.35\%) & Reference \\
\hline N2 & 226 (36.16\%) & 193 (36.69\%) & $33(33.33 \%)$ & 0.335 \\
\hline N3 & $101(16.16 \%)$ & $70(13.31 \%)$ & 31 (31.31\%) & $<0.001$ \\
\hline \multicolumn{5}{|l|}{ M stage } \\
\hline Mo & 561 (89.76\%) & 482 (91.63\%) & 79 (79.80\%) & $<0.001$ \\
\hline M1 & 64 (10.24\%) & $44(8.37 \%)$ & 20 (20.20\%) & \\
\hline \multicolumn{5}{|c|}{ Radiotherapy } \\
\hline No & $62(9.92 \%)$ & 46 (8.75\%) & $16(16.16 \%)$ & 0.024 \\
\hline Yes & $563(90.08 \%)$ & 480 (91.25\%) & 83 (83.84\%) & \\
\hline \multicolumn{5}{|c|}{ Chemotherapy } \\
\hline No & $61(9.76 \%)$ & 49 (9.32\%) & 12 (12.12\%) & 0.388 \\
\hline Yes & 564 (90.24\%) & 477 (90.68\%) & 87 (87.88\%) & \\
\hline
\end{tabular}

ECS, extracapsular spread; WHO, World Health Organization; AJCC, the American Joint Committee on Cancer.

24-113), 45 months (IQR, 8-109), and 88 months (IQR, 30-114) for the whole group, ECS group, and non-ECS group, respectively. All patients were diagnosed before 2010 . Consequently, the 6th edition AJCC staging system was applied to the patients in this study.

\section{Associated Factors of ECS}

In the logistic regression analysis, ECS was only associated with AJCC stage (Figure 2). ECS was more likely to present in stage IVb [odds ratio $(\mathrm{OR})=3.29 ; 95 \% \mathrm{CI}, 1.61-6.67 ; P=0.001$ ] and stage IVc $(\mathrm{OR}=4.22 ; 95 \% \mathrm{CI}, 2.02-8.90 ; P<0.001)$ than in stage 


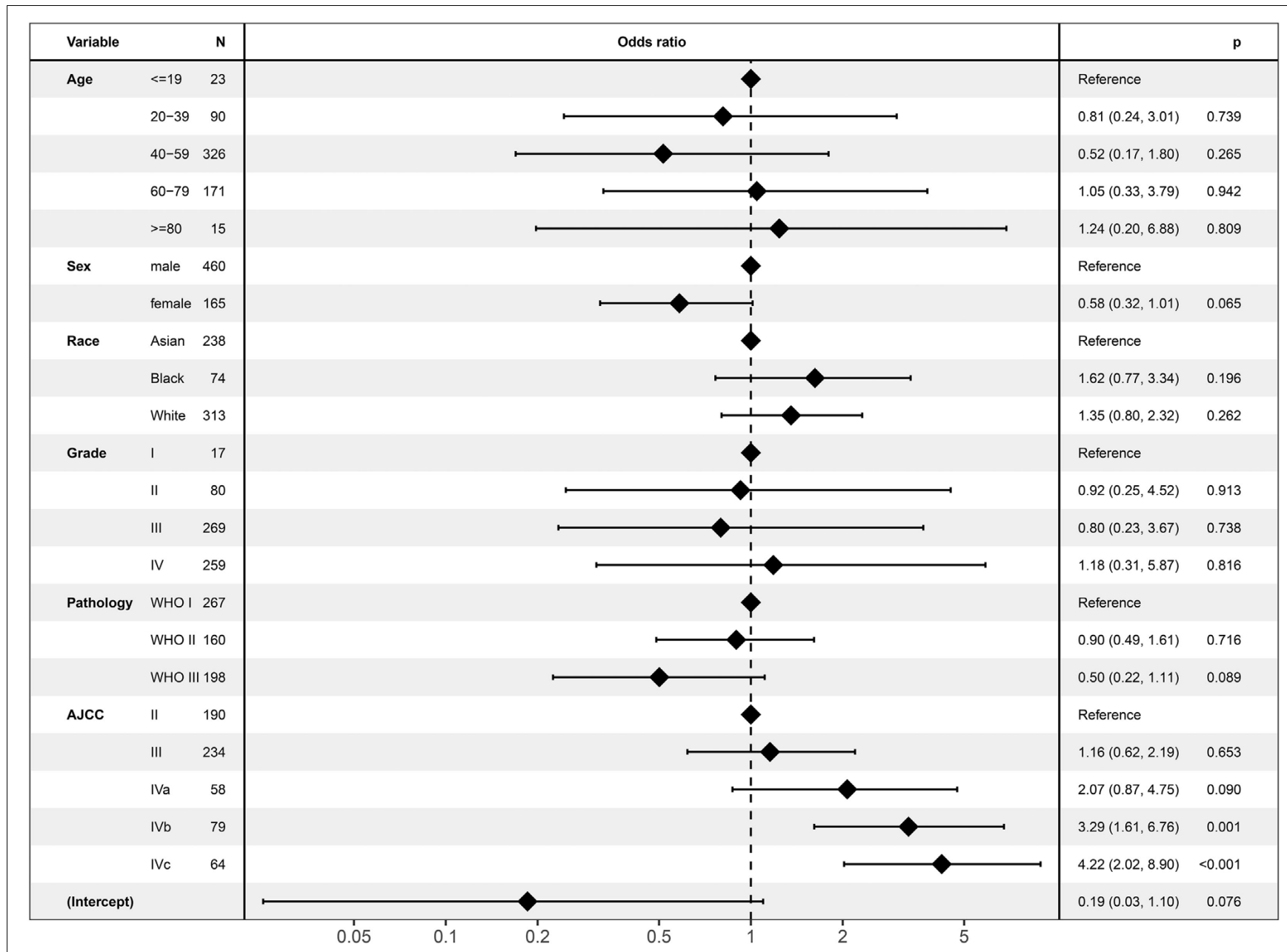

FIGURE 2 | Logistic regression analysis for associated variables of extracapsular spread.
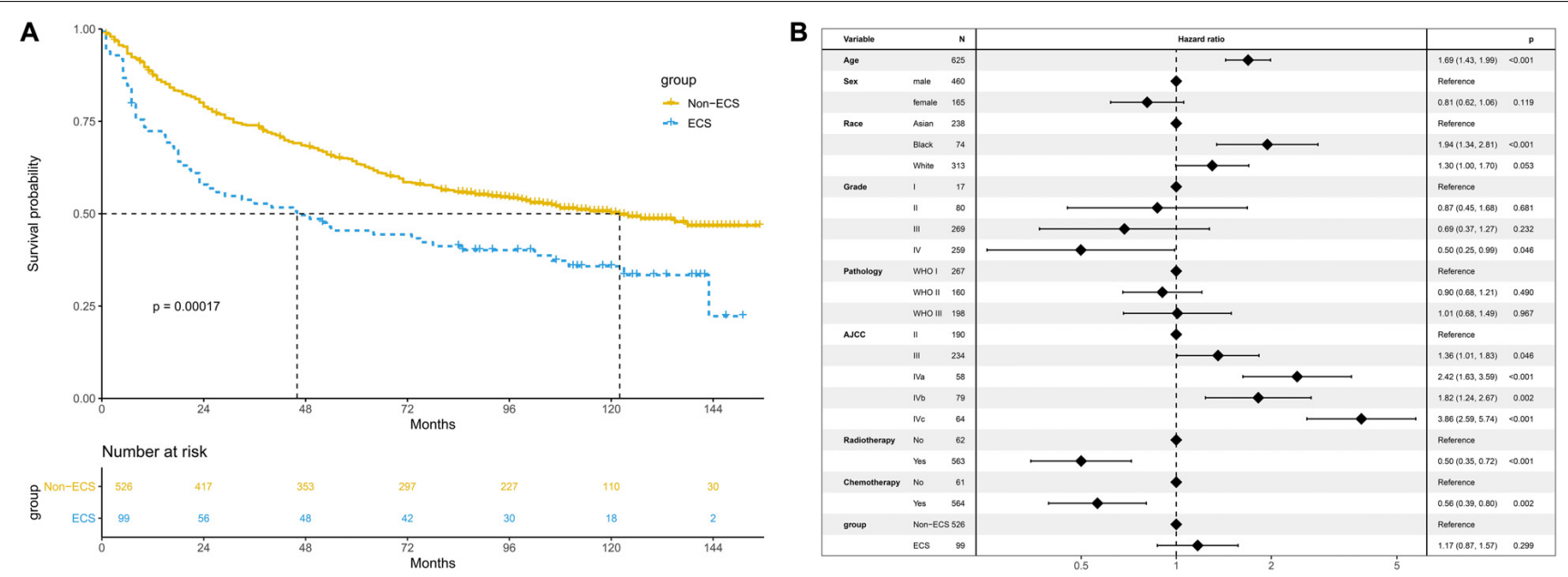

FIGURE 3 | Prognosis of ECS for OS in the unmatched cohort. (A) OS between the ECS and non-ECS groups. (B) Multivariable regression analysis of prognostic factors for OS. ECS, extracapsular spread; OS, overall survival. 

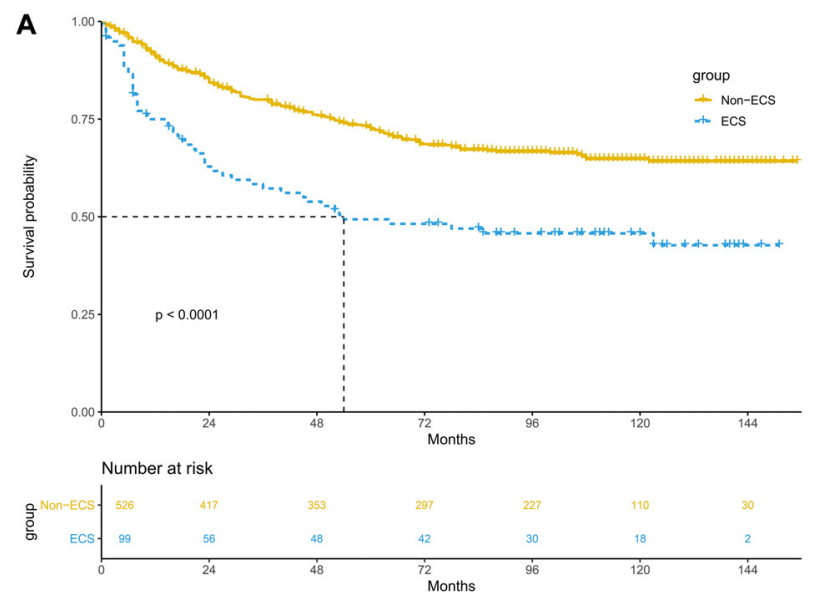

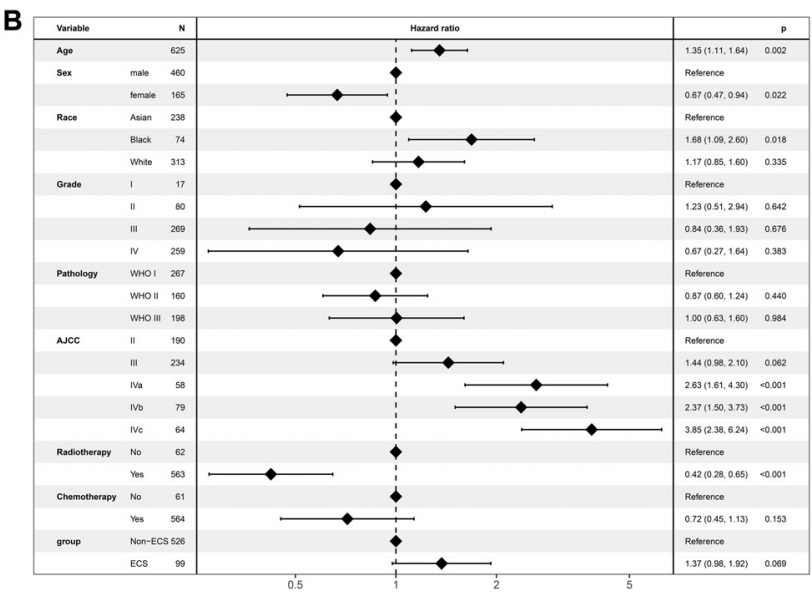

FIGURE 4 | Prognosis of ECS for CSS in the unmatched cohort. (A) CSS between the ECS and non-ECS groups. (B) Multivariable regression analysis of prognostic factors for CSS. ECS, extracapsular spread; CSS, cancer-specific survival.
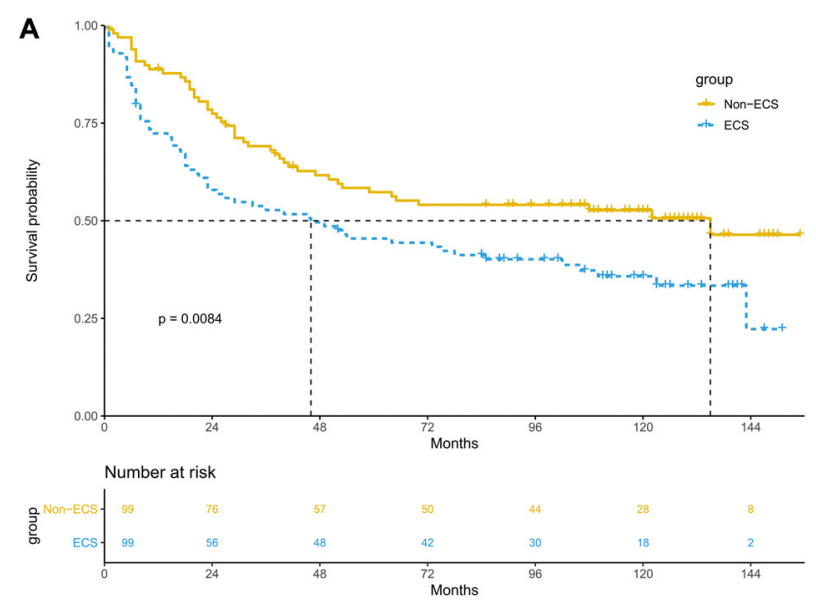

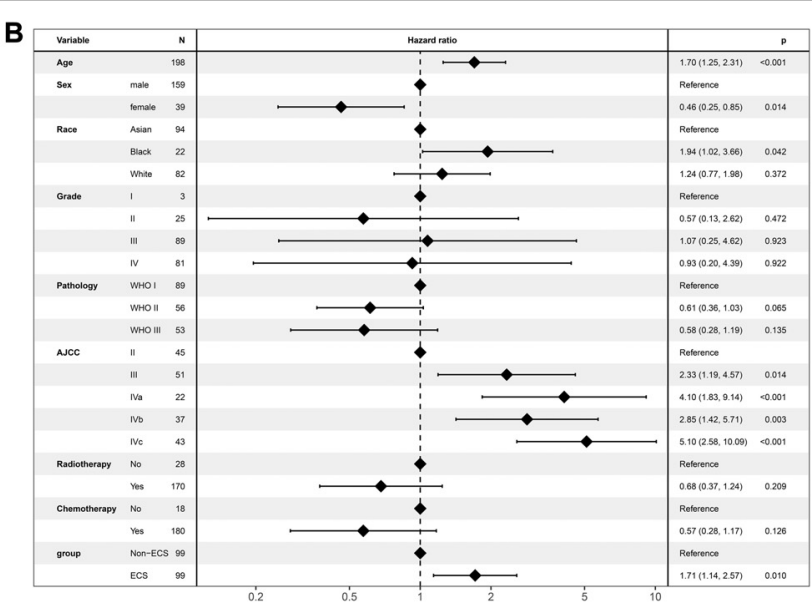

FIGURE 5 | Prognosis of ECS for OS in the propensity-matched cohort. (A) OS between the ECS and non-ECS groups. (B) Multivariable regression analysis of prognostic factors for OS. ECS, extracapsular spread; OS, overall survival.

II NPC. However, ECS status was not different between stages II, III, and IVa. ECS was not correlated with age, sex, race, tumor grade, or pathology.

\section{Survival Before PSM}

The 10-year OS of the non-ECS group was better than that of the ECS group (50.2 vs. $35.8 \%, P<0.001$; Figure 3A). However, ECS was not an independent prognostic factor for $\mathrm{OS}(\mathrm{HR}=1.17 ; 95 \%$ CI, 0.87-1.57; $P=0.299$; Figure 3B).

The non-ECS group showed a more favorable 10-year CSS than the ECS group (64.8 vs. 45.7\%, $P<0.001$; Figure 4A). However, ECS was not an independent risk prognostic factor for CSS $(\mathrm{HR}=1.37 ; 95 \% \mathrm{CI}, 0.98-1.92 ; P=0.069$; Figure 4B).

\section{Survival After PSM}

After PSM, 99 patients with non-ECS and 99 patients with ECS were matched. In the matched cohort, the ECS group showed a worse 10 -year OS than the non-ECS group (35.8 vs. 52.7\%; $P=0.008$; Figure 5A). In the multivariable regression analysis, ECS was an independent risk prognostic factor for OS $(\mathrm{HR}=1.71$; 95\% CI, 1.14-2.57; $P=0.010$; Figure 5B).

The 10-year CSS of the ECS group was worse than that of the non-ECS group (45.7 vs. $61.2 \% ; P=0.008$; Figure 6A). In the multivariable regression analysis, ECS was an independent risk prognostic factor for CSS (HR $=1.91 ; 95 \% \mathrm{CI}, 1.21-3.01$; $P=0.005$; Figure 6B).

\section{DISCUSSION}

This study revealed that patients with NPC with ECS of cervical lymph node metastases had worse OS and CSS compared with patients without ECS. ECS was a poor prognostic factor of NPC. The results indicated that the presence of ECS reflected aggressive biological behavior of NPC. Patients with ECS of cervical lymph 

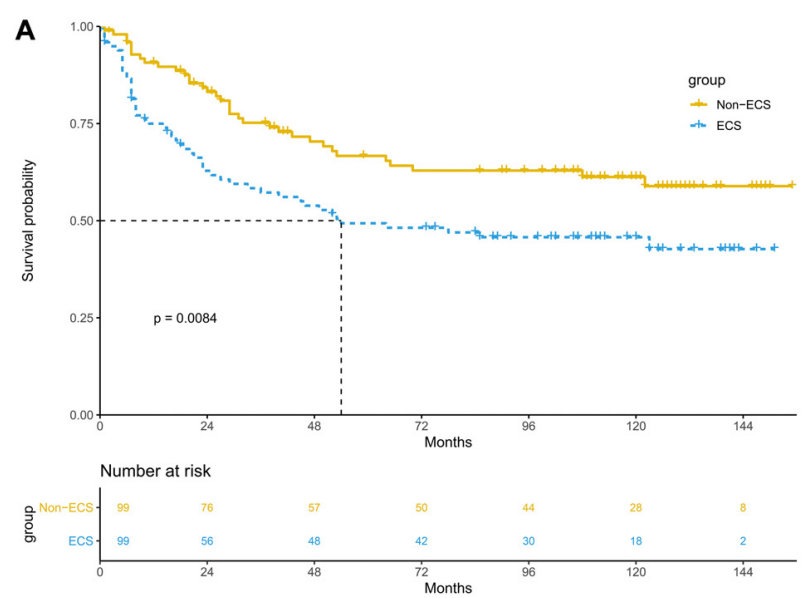

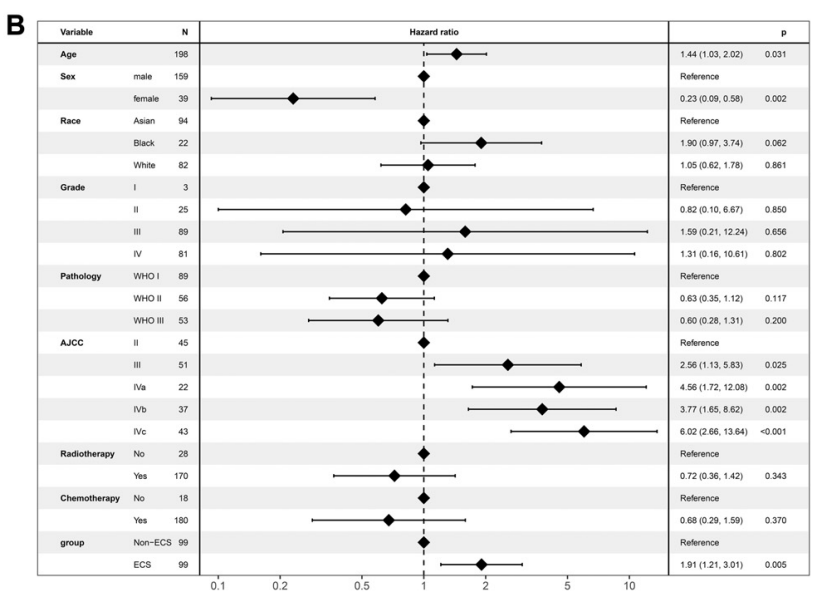

FIGURE 6 | Prognosis of ECS for CSS in the propensity-matched cohort. (A) CSS between the ECS and non-ECS groups. (B) Multivariable regression analysis of prognostic factors for CSS. ECS, extracapsular spread; CSS, cancer-specific survival.

node metastases might require more intensive treatments to achieve better survival.

Extracapsular spread was not included in the 8th edition of the AJCC staging system for two reasons (16). First, the detection accuracy of ECS should be improved. The gold standard of diagnosis for ECS is pathology. However, the ECS of cervical lymph node metastases in NPC is mainly assessed by image information, which is unable to reflect the true ECS status of cervical lymph node metastases detected by pathology. Previous studies focused on the overall accuracy of magnetic resonance imaging for detecting ECS revealed a specificity of $72-78 \%$, a sensitivity of $74-80 \%$, and an accuracy of $76-86 \%(17,18)$. On the other hand, ECS in NPC is subjective and might lead to a wider variation in interpretation. Interobserver agreement showed substantial difference in the identification of ECS, varying from 33.6 to $75.6 \%$ among studies (4-6).

Second, several studies have suggested that ECS is not a prognostic factor. Guo et al. (6) found that no significant prognostic values for ECS were found in terms of distant metastasis-free survival $(P=0.264)$, regional relapse-free survival $(P=0.931)$, and OS $(P=0.629)$. Similarly, Li et al. (5) revealed that regional failure $(\mathrm{HR}=1.41 ; 95 \% \mathrm{CI}, 0.47-4.22 ; P=0.54)$, distant failure $(\mathrm{HR}=1.27 ; 95 \% \mathrm{CI}, 0.83-1.95 ; P=0.26)$, and disease failure $(\mathrm{HR}=1.35 ; 95 \% \mathrm{CI}, 0.94-1.95 ; P=0.11)$ were not different between patients with ECS and patients without ECS.

In contrast, Mao et al. (4) reported that ECS was an independent prognostic factor for distant failure $(P<0.01)$ and disease failure $(P<0.01)$. Similarly, the current study also revealed that ECS was associated with a poor OS and CSS. The different results between these studies might be attributed to the different radiotherapy techniques used. In the study of Mao et al. (4), $83.7 \%$ of patients were treated with two-dimensional conventional radiotherapy (2D-CRT), $12.7 \%$ of patients were treated with intensity-modulated radiation therapy (IMRT), and $3.6 \%$ of patients received three-dimensional radiotherapy. In contrast, the patients in the other two studies were all treated with IMRT. It was reported that IMRT improves survival compared with 2D-CRT $(19,20)$. Thus, it is possible that patients with ECS receiving IMRT might achieve similar survival as patients without $\operatorname{ECS}(5,6)$.

However, the efficacy of radiotherapy on survival is not well identified for patients with NPC. Although IMRT was reported to be superior to 2D-CRT $(19,20)$, several studies have revealed that no difference was observed in OS between IMRT and 2DCRT $(21,22)$. Given the limitations of the SEER database, the radiotherapy technique could not be extracted in this study. Thus, we could not assess the impact of radiotherapy techniques on survival. Further studies should be performed to verify the results in the era of IMRT.

Extracapsular spread might be a factor that indicates aggressive biological behavior. The logistic regression analysis in the current study revealed that ECS was only associated with AJCC stage. Compared with that of non-ECS, the incidence of ECS was higher in stages IVb and IVc diseases. Similarly, Mao et al. (4) reported that nodal size was statistically correlated with ECS $(P<0.001)$. ECS was more likely to present in large lymph nodes. These results might suggest that NPC tumor cells rapidly metastasize into cervical lymph nodes, which leads to ECS. Then, these tumor cells could metastasize to distant locations.

Pathology of cervical lymph node metastases in NPC is not available in clinical practice. The assessment of ECS is mainly based on physical examination and imaging instead of pathology. Lymph nodes with ECS might invade the skin, adjacent soft tissues, or nerves. These signs could be found by physical examination. In imaging, lymph nodes presenting indistinct margins, irregular nodal capsular enhancement, infiltration into the adjacent tissues, or fusion status could be diagnosed as ECS (11-13). Moreover, no significant difference of sensitivity $(P=0.1317)$ and specificity $(P=0.3173)$ for the identification of ECS using MRI and CT were observed (17). In the SEER database, methods of ECS assessment could not be extracted. As a result, this study could not identify the sample sizes of ECS diagnosed with MRI, CT scan, or physical examination. This limitation might influence the OS and CSS in this study. On the other 
hand, prognostic value of ECS based on imaging in patients with NPC was inconsistent between previous studies (4-6, 9). Thus, further studies with large sample sizes are needed to verify the prognosis of ECS in NPC.

This study had some limitations. First, data on localregional failure and distant failure were not available due to the limitations of the SEER database. The impact of ECS on local-regional-free survival and distant metastasis-free survival could not be assessed. It is important to develop treatments for patients with ECS in clinical practice. Second, selection biases inherently existed in this retrospective cohort study, which made the intrinsic quality of the data poor. To control potential biases, we performed several analytic techniques, including multivariable adjustment and PSM. The multivariable analysis in the matched cohort revealed that ECS was an independent risk factor for OS and CSS.

\section{CONCLUSION}

This study indicated that ECS is a prognostic risk factor of NPC. Due to the limitations of the SEER database and retrospective nature of this study, the results should be treated with caution.

\section{REFERENCES}

1. Chen W, Zheng R, Baade PD, Zhang S, Zeng H, Bray F, et al. Cancer statistics in China, 2015. CA Cancer J Clin. (2016) 66:115-32. doi: 10.3322/caac.21338

2. Siegel RL, Miller KD, Jemal A. Cancer statistics, 2018. CA Cancer J Clin. (2018) 68:7-30. doi: $10.3322 /$ caac. 21442

3. Wang X, Hu C, Ying H, He X, Zhu G, Kong L, et al. Patterns of lymph node metastasis from nasopharyngeal carcinoma based on the 2013 updated consensus guidelines for neck node levels. Radiother Oncol. (2015) 115:41-5. doi: 10.1016/j.radonc.2015.02.017

4. Mao YP, Liang SB, Liu LZ, Chen Y, Sun Y, Tang LL, et al. The N staging system in nasopharyngeal carcinoma with radiation therapy oncology group guidelines for lymph node levels based on magnetic resonance imaging. Clin Cancer Res. (2008) 14:7497-503. doi: 10.1158/1078-0432.CCR-08-0271

5. Li WF, Sun Y, Mao YP, Chen L, Chen YY, Chen M, et al. Proposed lymph node staging system using the International Consensus Guidelines for lymph node levels is predictive for nasopharyngeal carcinoma patients from endemic areas treated with intensity modulated radiation therapy. Int J Radiation Oncol Biol Phys. (2013) 86:249-56. doi: 10.1016/j.ijrobp.2012.09.003

6. Guo Q, Pan J, Zong J, Zheng W, Zhang C, Tang L, et al. Suggestions for lymph node classification of UICC/AJCC staging system: a retrospective study based on 1197 nasopharyngeal carcinoma patients treated with intensitymodulated radiation therapy. Medicine. (2015) 94:e808. doi: 10.1097/MD. 0000000000000808

7. Greenberg JS, Fowler R, Gomez J, Mo V, Roberts D, El Naggar AK, et al. Extent of extracapsular spread: a critical prognosticator in oral tongue cancer. Cancer. (2003) 97:1464-70. doi: 10.1002/cncr.11202

8. Hirabayashi H, Koshii K, Uno K, Ohgaki H, Nakasone Y, Fujisawa T, et al. Extracapsular spread of squamous cell carcinoma in neck lymph nodes: prognostic factor of laryngeal cancer. Laryngoscope. (1991) 101:502-6. doi: 10.1288/00005537-199105000-00010

9. Kang M, Zhou P, Wei T, Zhao T, Long J, Li G, et al. A novel N staging system for NPC based on IMRT and RTOG guidelines for lymph node levels: results of a prospective multicentric clinical study. Oncol Lett. (2018) 16:308-16. doi: $10.3892 /$ ol.2018.8676

10. Edge SB, Compton CC. The American joint committee on cancer: the 7th edition of the AJCC cancer staging manual and the future of TNM. Ann Surg Oncol. (2010) 17:1471-4. doi: 10.1245/s10434-010-0985-4

\section{DATA AVAILABILITY STATEMENT}

The datasets generated for this study are available on request to the corresponding author.

\section{AUTHOR CONTRIBUTIONS}

X-BP contributed to the conception of the study. XY and LL performed the data analyses, contributed to manuscript preparation, and helped to perform the analysis with constructive discussions. All authors contributed to the article and approved the submitted version.

\section{FUNDING}

This study was supported by a grant from the Department of Education of the Guangxi Zhuang Autonomous Region (No. KY2016LX029), Guangxi Medical University (No. GXMUYSF201521), and the Research and Development Project of Guangxi (Nos. 1598012-22 and AB18221007).

11. Pan J, Xu Y, Qiu S, Zong J, Guo Q, Zhang Y, et al. A comparison between the Chinese 2008 and the 7th edition AJCC staging systems for nasopharyngeal carcinoma. Am J Clin Oncol. (2015) 38:189-96. doi: 10.1097/ COC.0b013e31828f5c96

12. Tang L, Li L, Mao Y, Liu L, Liang S, Chen Y, et al. Retropharyngeal lymph node metastasis in nasopharyngeal carcinoma detected by magnetic resonance imaging : prognostic value and staging categories. Cancer. (2008) 113:347-54. doi: $10.1002 / \mathrm{cncr} .23555$

13. van den Brekel MW, Stel HV, Castelijns JA, Nauta JJ, van der Waal I, Valk J, et al. Cervical lymph node metastasis: assessment of radiologic criteria. Radiology. (1990) 177:379-84. doi: 10.1148/radiology.177.2.2217772

14. Wu SG, Liao XL, He ZY, Tang LY, Chen XT, Wang Y, et al. Demographic and clinicopathological characteristics of nasopharyngeal carcinoma and survival outcomes according to age at diagnosis: a population-based analysis. Oral Oncol. (2017) 73:83-7. doi: 10.1016/j.oraloncology.2017.08.006

15. Austin PC. Optimal caliper widths for propensity-score matching when estimating differences in means and differences in proportions in observational studies. Pharm Stat. (2011) 10:150-61. doi: 10.1002/pst.433

16. Pan JJ, Ng WT, Zong JF, Chan LL, O'Sullivan B, Lin SJ, et al. Proposal for the 8th edition of the AJCC/UICC staging system for nasopharyngeal cancer in the era of intensity-modulated radiotherapy. Cancer. (2016) 122:546-58. doi: 10.1002/cncr.29795

17. King AD, Tse GM, Yuen EH, To EW, Vlantis AC, Zee B, et al. Comparison of CT and MR imaging for the detection of extranodal neoplastic spread in metastatic neck nodes. Eur J Radiol. (2004) 52:264-70. doi: 10.1016/j.ejrad. 2004.03.004

18. Steinkamp HJ, Beck A, Werk M, Felix R. [Extracapsular spread of cervical lymph node metastases: diagnostic value of magnetic resonance imaging]. Rofo. (2002) 174:50-5.

19. Zhang B, Mo Z, Du W, Wang Y, Liu L, Wei Y. Intensity-modulated radiation therapy versus 2D-RT or 3D-CRT for the treatment of nasopharyngeal carcinoma: a systematic review and meta-analysis. Oral Oncol. (2015) 51:10416. doi: 10.1016/j.oraloncology.2015.08.005

20. Peng G, Wang T, Yang KY, Zhang S, Zhang T, Li Q, et al. A prospective, randomized study comparing outcomes and toxicities of intensity-modulated radiotherapy vs. conventional two-dimensional radiotherapy for the treatment of nasopharyngeal carcinoma. Radiother Oncol. (2012) 104:286-93. doi: 10. 1016/j.radonc.2012.08.013 
21. Marta GN, Silva V, de Andrade Carvalho H, de Arruda FF, Hanna SA, Gadia $\mathrm{R}$, et al. Intensity-modulated radiation therapy for head and neck cancer: systematic review and meta-analysis. Radiother Oncol. (2014) 110:9-15. doi: 10.1016/j.radonc.2013.11.010

22. Zhang MX, Li J, Shen GP, Zou X, Xu JJ, Jiang R, et al. Intensitymodulated radiotherapy prolongs the survival of patients with nasopharyngeal carcinoma compared with conventional two-dimensional radiotherapy: a 10-year experience with a large cohort and long follow-up. Eur J Cancer. (2015) 51:2587-95. doi: 10.1016/j.ejca.2015. 08.006
Conflict of Interest: The authors declare that the research was conducted in the absence of any commercial or financial relationships that could be construed as a potential conflict of interest.

Copyright $\odot 2020$ Yin, Lv and Pan. This is an open-access article distributed under the terms of the Creative Commons Attribution License (CC BY). The use, distribution or reproduction in other forums is permitted, provided the original author(s) and the copyright owner(s) are credited and that the original publication in this journal is cited, in accordance with accepted academic practice. No use, distribution or reproduction is permitted which does not comply with these terms. 